\title{
Correction to: Role of PUMA in the methamphetamine-induced migration of microglia
}

\author{
Lei Zhao ${ }^{1} \cdot{\text { Longfei } \mathrm{Du}^{1} \cdot \text { Yanhong Zhang }}^{1} \cdot$ Jie $\mathrm{Chao}^{2} \cdot$ Ming Duan $^{3} \cdot$ Honghong Yao $^{1,4} \cdot$ Chuanlu Shen $^{5}$. \\ Yuan Zhang ${ }^{1}$
}

Published online: 29 May 2020

(C) Springer Science+Business Media, LLC, part of Springer Nature 2020

\section{Correction to: Metabolic Brain Disease 34 (1): 61 - 69} https://doi.org/10.1007/s11011-018-0319-y

In the original publication of this article, wrong western blot images were inadvertently included in Figs. 2 and 3. The corrected figures are shown below. The authors declare that these amendments do not change the results or conclusions of their paper, and apologize for this oversight.

Publisher's note Springer Nature remains neutral with regard to jurisdictional claims in published maps and institutional affiliations.

The online version of the original article can be found at https://doi.org/ 10.1007/s11011-018-0319-y

Yuan Zhang

yuanzhang@seu.edu.cn

1 Department of Pharmacology, Medical School of Southeast University, Southeast University, Nanjing 210009, Jiangsu, China

2 Department of Physiology, Medical School of Southeast University, Southeast University, Nanjing, China

3 Key Laboratory for Zoonosis Research, Ministry of Education, Jilin University, Changchun, China

4 Institute of Life Sciences, Key Laboratory of Developmental Genes and Human Disease, Southeast University, Nanjing, China

5 Department of Pathophysiology, Medical School of Southeast University, Southeast University, Nanjing, China 
a
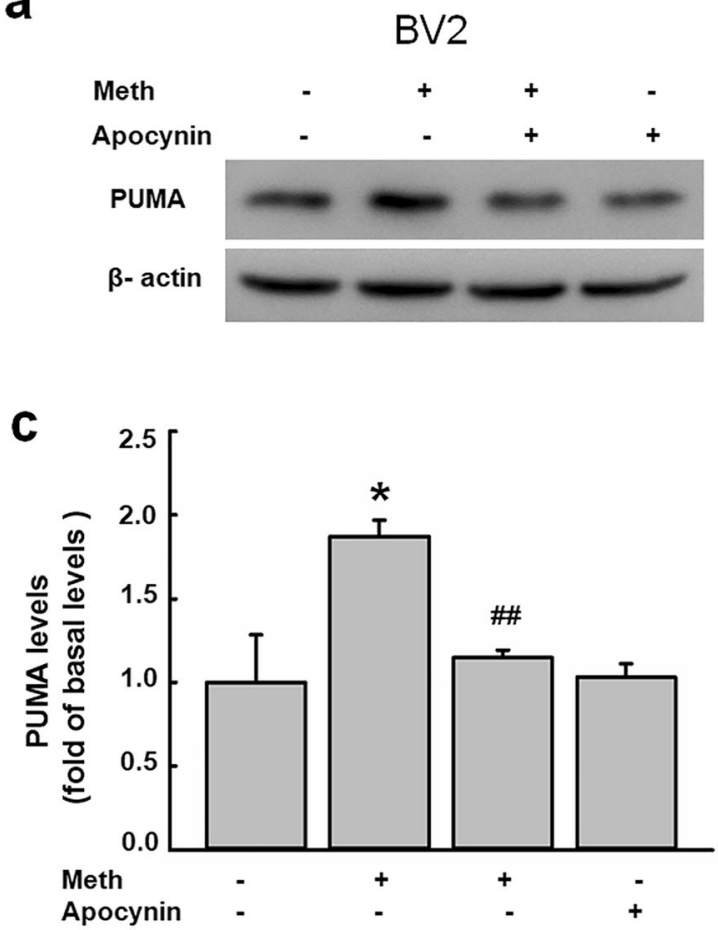

Fig. 2 ROS was involved in the methamphetamine-induced upregulation of PUMA in microglia. a, b Representative western blot showing the effects of methamphetamine on the increase in PUMA expression, which was attenuated by a ROS inhibitor (apocynin, $10 \mu \mathrm{M}$ ) in BV2 and HAPI cells. c, d Densitometric analyses suggested that b

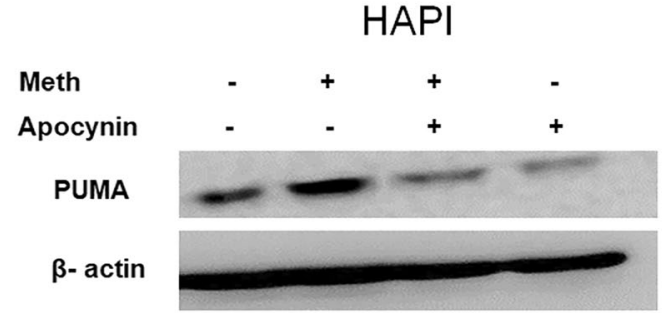

d

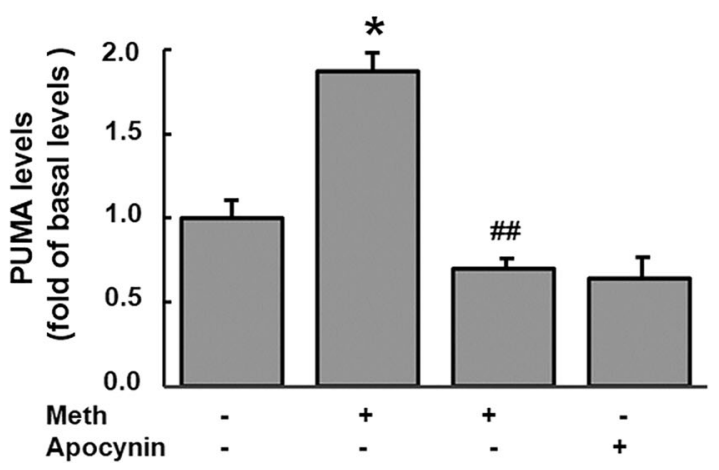

methamphetamine increased PUMA expression, which was attenuated by the apocynin pretreatment. $* p<0.05, * * p<0.01$ vs the control group; \#\# $p<0.01$ vs the methamphetamine-treated group using oneway ANOVA. Meth, methamphetamine 
a

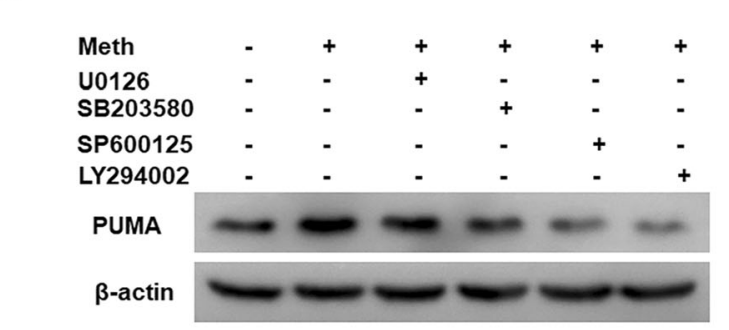

C

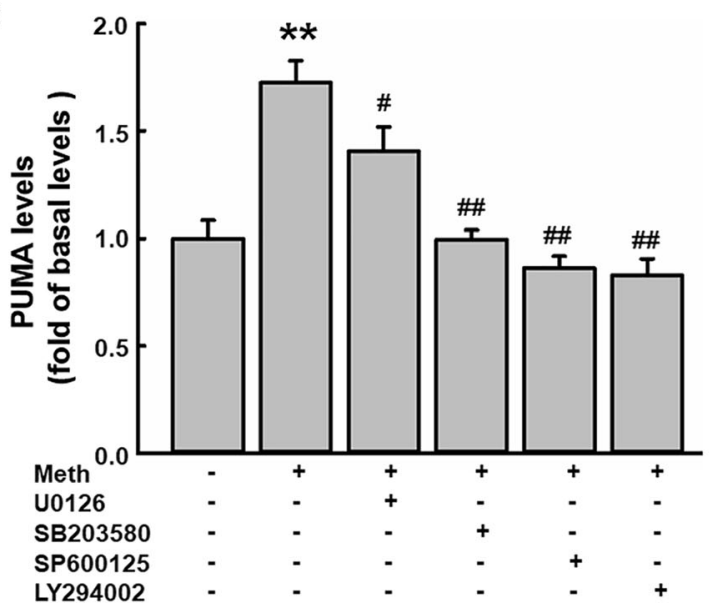

Fig. 3 The MAPK and PI3K/Akt pathways were involved in the methamphetamine-induced up-regulation of PUMA in microglia. $\mathbf{a}, \mathbf{b}$ Representative western blot showing the effects of methamphetamine on the increase in PUMA expression, which was attenuated by the MAPK inhibitor (SB203580, $10 \mu \mathrm{M}$ ), the JNK inhibitor (SP600125, $10 \mu \mathrm{M}$ ) and the PI3K/Akt inhibitor (LY294002, $10 \mu \mathrm{M}$ ) but not the b HAPI

$\begin{array}{lllllll}\text { Meth } & - & + & + & + & + & + \\ \text { U0126 } & - & - & + & - & - & - \\ \text { SB203580 } & - & - & - & + & - & - \\ \text { SP600125 } & - & - & - & - & + & - \\ \text { LY294002 } & - & - & - & - & - & +\end{array}$

PUMA

$\beta$-actin

d

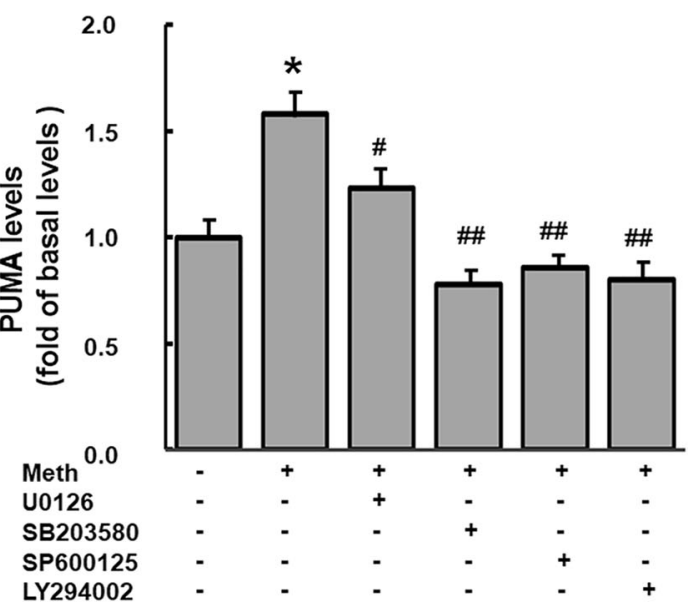

ERK inhibitor (U0126, $10 \mu \mathrm{M})$ in BV2 and HAPI cells. c, d Densitometric analyses suggested that methamphetamine increased PUMA expression, which was attenuated by the SB203580, SP600125 and LY294002 pretreatments but not the U0126 pretreatment. * $p<0.05$, ** $p<0.01$ vs the control group; \#\# $p<0.01$ vs the methamphetaminetreated group using one-way ANOVA. Meth, methamphetamine 